\title{
Dark-blood late gadolinium-enhancement cardiac magnetic resonance imaging for myocardial scar detection based on simplified timing scheme: single-center experience in patients with suspected coronary artery disease
}

\author{
Rungroj Krittayaphong $^{1 \#}$, Shuo Zhang ${ }^{2,3 \#}$, Prajak Tanapibunpon ${ }^{4}$, Yodying Kaolawanich ${ }^{1}$, Supaporn Nakyen ${ }^{4}$ \\ ${ }^{1}$ Division of Cardiology, Department of Medicine, Faculty of Medicine Siriraj Hospital, Mahidol University, Bangkok, Thailand; ${ }^{2}$ Philips Healthcare, \\ Singapore; ${ }^{3}$ Philips Healthcare, Hamburg, Germany; ${ }^{4}$ Her Majesty Cardiac Center, Faculty of Medicine Siriraj Hospital, Mahidol University, \\ Bangkok, Thailand
}

Contributions: (I) Conception and design: R Krittayaphong, S Zhang, P Tanapibunpon; (II) Administrative support: R Krittayaphong; (III) Provision of study materials or patients: R Krittayaphong, P Tanapibunpon, Y Kaolawanich, S Nakyen; (IV) Collection and assembly of data: R Krittayaphong, P Tanapibunpon, Y Kaolawanich, S Nakyen; (V) Data analysis and interpretation: R Krittayaphong, S Zhang, P Tanapibunpon, Y Kaolawanich, S Nakyen; (VI) Manuscript writing: All authors; (VII) Final approval of manuscript: All authors.

"These authors contributed equally to this work.

Correspondence to: Rungroj Krittayaphong, MD. Division of Cardiology, Department of Medicine, Faculty of Medicine Siriraj Hospital, Mahidol University, 2 Wanglang Road, Bangkoknoi, Bangkok 10700, Thailand. Email: rungroj.kri@mahidol.ac.th.

Background: This study aims to examine scar detectability using dark-blood late gadolinium enhancement (LGE) with simplified timing scheme and fixed parameters comparing to two conventional bright-blood approaches in patients with known or suspected coronary artery disease.

Methods: Three LGE techniques were performed in all patients with known or suspected coronary artery disease at $3 \mathrm{~T}$ : dark blood two-dimensional (2D) phase-sensitive inversion recovery (PSIR) preceded with a T2-preparation pulse (DB-LGE), conventional three-dimensional (3D) gradient-echo inversion recovery (3D-IR) and conventional 2D PSIR. Timing parameters in DB-LGE were tested in five clinically confirmed coronary artery disease patients with scars and fixed for the rest of the study. Two independent readers evaluated images at both patient and segment levels. Image quality and contrast ratio between scar and adjacent tissues were assessed. Concordance between the three techniques and detection rate based on expert consensus were reported.

Results: Forty-six patients were recruited in the study (average age 66.8 years, $69.6 \%$ male). DB-LGE demonstrated superior image quality $(\mathrm{P}=0.001$ vs. 3D-IR) and scar-to-blood contrast ratio $(\mathrm{P}<0.001$ vs. 3DIR and PSIR). Among 41 patients with suspected coronary artery disease, myocardial scar was present in 30 patients (73.2\%), all detected by DB-LGE, yielding a detection rate of $100 \%$ compared to $93.3 \%$ and $96.7 \%$ for bright-blood 3D-IR and PSIR. For subendocardial scar detection among 656 segments, DB-LGE had a detection rate of $99.4 \%$ compared to $57.8 \%$ for $3 \mathrm{D}$-IR and $61.0 \%$ for PSIR (both $\mathrm{P}<0.001$ ).

Conclusions: DB-LGE improves detection of myocardial scar compared with conventional bright-blood LGE techniques, particularly of subendocardial scar.

Keywords: Coronary artery disease (CAD); late gadolinium enhancement (LGE); myocardial infarction; dark blood scar imaging; phase-sensitive inversion recovery (PSIR) 
Submitted Jul 07, 2021. Accepted for publication Oct 08, 2021.

doi: 10.21037/qims-21-704

View this article at: https://dx.doi.org/10.21037/qims-21-704

\section{Introduction}

Detection and assessment of myocardial scar is crucial in diagnosis, prognosis and treatment guidance in patients with suspected or known coronary artery disease (CAD) (1-5). Cardiac magnetic resonance (CMR) by using late gadolinium enhancement (LGE) is considered the standard for scar detection $(6,7)$, presenting a hyperenhancement pattern to be distinguished from viable myocardium. Despite various efforts to improve the acquisition technique for spatial resolution or coverage $(8,9)$, one important limitation of LGE CMR is that myocardial scar and ventricular blood pool are often hyperenhanced to a similar degree following administration of contrast agent $(6,7)$. This may lead to poor delineation and interpretation of infarcted myocardium, particularly in the subendocardial area, as it is immediately adjacent to the bright left ventricle (LV) cavity (10).

Recent technical advances in dark-blood (DB) LGE CMR provide improved tissue delineation with signal suppression of the blood pool based on $\mathrm{T} 2$-preparation pulse that is relatively independent from the blood flow velocities (11-15). However, they require additional scout scans to visually or manually determine the optimal imaging parameters for the DB sequence. In addition, these new techniques are not generally available on all clinical systems, and all DB-methods are currently not standard of care in routine examinations.

In this study, we sought to examine scar detectability using DB-LGE in comparison to conventional bright-blood methods in patients with suspected $\mathrm{CAD}$, and to further verify the principle on $3 \mathrm{~T}$ with simplified timing scheme based on fixed parameters for accommodation in a clinical setup.

We present the following article in accordance with the STARD reporting checklist (available at https://dx.doi. org/10.21037/qims-21-704).

\section{Methods}

\section{Study population}

The study population comprised consecutive patients older than 18 years with known or suspected coronary artery disease who were referred for stress CMR. Additional inclusion criteria included: (I) history of previous myocardial infarction; (II) abnormal left ventricular wall motion from previous echocardiogram or CMR; (III) left ventricular ejection fraction (LVEF) less than $50 \%$ from previous echocardiogram or CMR. Patients who presented at least one of these conditions were recruited to increase the chance of having myocardial scar. We excluded patients with claustrophobia and those who could not complete CMR examination. This study was conducted in accordance with the Declaration of Helsinki (as revised in 2013). This study was approved by the Siriraj Institutional Review Board (SIRB) of the Faculty of Medicine Siriraj Hospital, Mahidol University, Bangkok, Thailand. All patients provided written informed consent prior to participation.

\section{CMR imaging}

All patients underwent CMR function, perfusion and LGE acquisitions at $3.0 \mathrm{~T}$ (Ingenia, Philips Healthcare, Best, The Netherlands) using standard torso and posterior receiver coils. For LGE imaging the previously introduced DB method (15) with two-dimensional (2D) phase-sensitive inversion recovery (PSIR) preceded by a T2-preparation (T2-prep) pulse was employed. A simplified timing scheme with $35 \mathrm{~ms}$ T2-prep echo time and $150 \mathrm{~ms}$ inversion delay time (TI) was adapted on $3 \mathrm{~T}$ according to literature reports $(16,17)$. This was first tested in five clinically confirmed CAD cases with documented scars (Figure 1) and was then fixed for the rest of the patient cohort. It was compared with two widely used bright-blood LGE scan protocols: conventional three-dimensional (3D) gradient-echo inversion recovery (3D-IR) that was routinely performed in our hospital, and conventional 2D PSIR. The imaging parameters are summarized in Table 1 . All three techniques were applied in a randomized order in the study population, and all LGE images were acquired at end diastole during breath-holding obtained 10 to 15 minutes after injection of $0.2 \mathrm{mmol} / \mathrm{kg}$ of gadobutrol (Gadovist, Bayer HealthCare Pharmaceuticals, Leverkusen, Germany) contrast medium.

\section{Qualitative assessment: scar detection and confidence reading}

CMR analysis was done in a blinded fashion to the clinical 


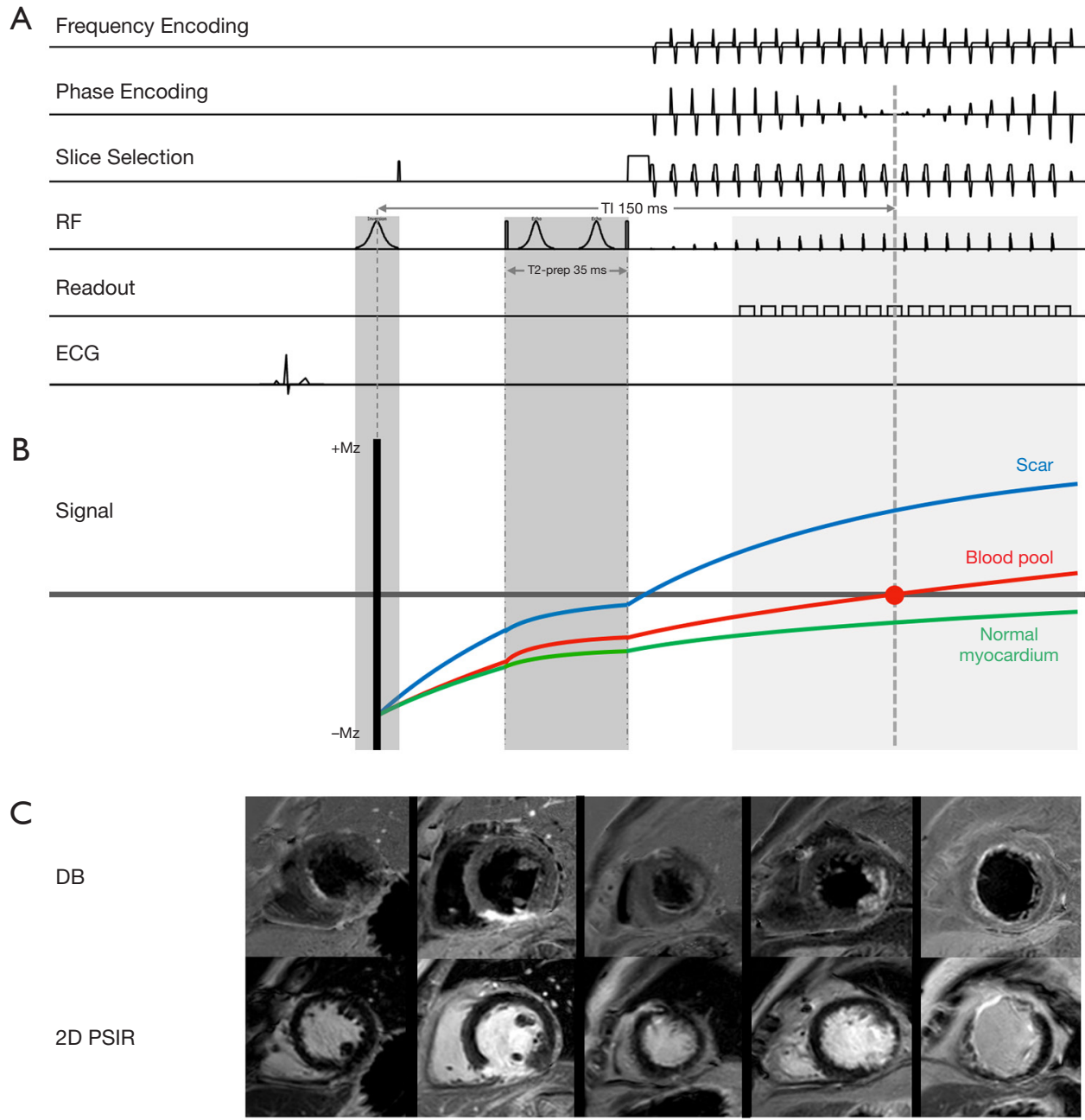

Figure 1 DB-LGE CMR method used in this study. (A) Imaging pulse sequence diagram of a phase-sensitive inversion recovery (PSIR) with a T2-preparation pulse (16,17). A simplified timing scheme with fixed parameter (T2-prep $35 \mathrm{~ms}$ and TI $150 \mathrm{~ms}$ ) were set according to five documented CAD patients with scars and fixed in the rest of the study. (B) Signal curve of DB-LGE showing the blood pool (red) is nulled according to the chosen timing scheme, whereas the scar signal (blue) has recovered due to much shortened T1. (C) DB-LGE images (above) obtained by using the aforementioned timing scheme and parameters with comparison to conventional PSIR (bottom) in five documented CAD patients. CAD, coronary artery disease; CMR, cardiac magnetic resonance; DB, dark-blood; LGE, late gadolinium enhancement.

findings by two readers (Y Kaolawanich and S Nakyen) with more than 10 years' experience in CMR. All three LGE methods were evaluated in a random order without knowing the findings of the other CMR techniques for (I) scar presence, (II) location, and (III) extent. Scar location refers to a per-segment analysis of its presence in the left anterior descending artery (LAD), left circumflex artery (LCX) or right coronary artery (RCA) territories based on a 17-segment model according to the American Heart Association (18), excluding the apical cap. Scar extent was assessed by the transmurality index with grade 1 (1-25\% of the wall), grade $2(26-50 \%)$, grade $3(51-75 \%)$ and grade 4 (76-100\%). Consequently, grade 1 and 2 were classified as non-transmural ( $\leq 50 \%$ ), while grade 3 and 4 as transmural (at least $51 \%$ ). For scar presence and transmurality, two independent readers performed first-line reading. In case of disagreement, it was resolved by a third reader $(\mathrm{R}$ Krittayaphong).

Confidence in scar detection and associated image quality was assessed using a 5 -point scale: $1=$ non-diagnostic with significant artifact, 2 = minimally diagnostic with strong artifact, 3 = diagnostic with moderate artifact, 4 = high 
Table 1 Imaging parameters of the LGE methods in the study

\begin{tabular}{|c|c|c|c|}
\hline Method & \multicolumn{2}{|c|}{ Conventional bright-blood LGE } & $\frac{\text { Dark-blood LGE }}{\text { DB }}$ \\
\hline Basic sequence & 3D gradient-echo IR & 2D PSIR & 2D T2-prepared PSIR \\
\hline $\mathrm{FOV}\left(\mathrm{mm}^{2}\right)$ & $270 \times 270$ & $270 \times 270$ & $270 \times 270$ \\
\hline Matrix size & $152 \times 148$ & $168 \times 207$ & $168 \times 207$ \\
\hline Slice thickness (ACQ) (mm) & 10.0 & 8.5 & 8.5 \\
\hline Slice thickness (REC) (mm) & 8.5 & - & - \\
\hline Slice gap (mm) & 0 & 0 & 0 \\
\hline \# of slices & 10 & 10 & 10 \\
\hline Flip angle (degree) & 15 & 25 & 25 \\
\hline Inversion time (TI) (ms) & $200-300$ & $300-400$ & 150 \\
\hline T2-prep TE (ms) & NA & NA & 35 \\
\hline Parallel imaging acceleration & SENSE 2.5 & SENSE 1.3 & SENSE 1.3 \\
\hline Breathhold time (s) & ap. 20 & ap. $16 \times 10$ & ap. $16 \times 10$ \\
\hline
\end{tabular}

ap., approximately; LGE, late gadolinium enhancement; IR, inversion recovery; PSIR, phase-sensitive inversion recovery; FOV, field of view; 3D, three-dimensional; 2D, two-dimensional; mm, millimeter; $A C Q$, acquired; REC, reconstructed; NA, not available.

diagnostic confidence with minimal artifact, $5=$ excellent diagnostic confidence with no artifact. CMR images were analyzed on the ISP workstation (IntelliSpace Portal 9.0, Philips Healthcare, Best, The Netherlands).

Consensus between all three readers was used as gold standard. This in combination of the per-segment analysis allowed comparative assessment taking scar location into account at both patient and segment levels for the presence or absence as well as the pattern of the myocardial scar. In addition, scar detection rate is defined as the rate of scars detected by each individual technique compared to the expert consensus as gold standard.

\section{Quantitative assessment: contrast ratio (CR)}

For quantitative scar analysis, regions of interest (ROIs) were manually drawn on slices, where scar was identified, in areas of scar hyperenhancement, a remote area of normal myocardium, and in the blood pool. For calculation of the signal ratio (SR) and CR, the manufacturer's applied scaling in the stored DICOM data was removed by converting the data to floating point values as this reflects the true magnetic resonance (MR) signal range directly after reconstruction. A linear intensity correction was applied to compensate for the negative value in the PSIR images (19) and relative SR was calculated referencing to the normal myocardium. For scar, $\left.S R_{\text {sar }}=\left[\left(S_{\text {sar }}+2,048\right)\right) /\left(S_{\text {myocardium }}+2,048\right)\right] \cdot 100-100$, where $S_{\text {scar }}$ indicated signal intensity of the ROI in arbitrary units. These were applied for all three LGE methods with no scaling for comparison purpose. Subsequently, CR was derived for the difference of the relative signal between scar and blood pool $\left(C R_{\text {sar-blood }}=S R_{\text {scar }}-S R_{\text {blood }}\right)$, between scar and normal myocardium $\left(\mathrm{CR}_{\text {scar-myo }}\right)$, and between blood and normal myocardium $\left(\mathrm{CR}_{\text {blood-myo }}\right)$.

\section{Statistical analysis}

Continuous data with normal distribution are presented as mean \pm standard deviation $(\mathrm{SD})$ and as median (interquartile range) with non-normal distribution. Discrete data are presented as number (percentage). Scar presence, confidence reading, general image quality was evaluated 
Table 2 Patient characteristics of the study cohort

\begin{tabular}{|c|c|}
\hline Variables & Mean \pm SD or $n(\%)$ \\
\hline \multicolumn{2}{|l|}{ Clinical characteristics } \\
\hline Age (years) & $66.8 \pm 10.2$ \\
\hline Male gender & $32(69.6)$ \\
\hline Weight (kg) & $69.2 \pm 10.4$ \\
\hline Height (cm) & $164.0 \pm 8.0$ \\
\hline Body mass index $\left(\mathrm{kg} / \mathrm{m}^{2}\right)$ & $25.8 \pm 4.1$ \\
\hline Hypertension & $28(60.9)$ \\
\hline Diabetes mellitus & $16(34.8)$ \\
\hline Hypercholesterolemia & $28(60.9)$ \\
\hline Smoking & $4(8.7)$ \\
\hline Prior myocardial infarction & $8(17.4)$ \\
\hline Prior revascularization & $10(21.7)$ \\
\hline History of chest pain & $16(34.8)$ \\
\hline History of dyspnea & $16(34.8)$ \\
\hline History of heart failure & $7(15.2)$ \\
\hline Beta-blockers & $23(50.0)$ \\
\hline Calcium antagonists & $8(17.4)$ \\
\hline Nitrates & $6(13.0)$ \\
\hline ACEI/ARB & $18(39.1)$ \\
\hline Antiplatelet & $20(43.5)$ \\
\hline Statins & $27(58.7)$ \\
\hline \multicolumn{2}{|l|}{ CMR variables } \\
\hline LVEF (\%) & $48.7 \pm 18.8$ \\
\hline LVEDVI $\left(\mathrm{mL} / \mathrm{m}^{2}\right)$ & $107.9 \pm 40.5$ \\
\hline LVESVI (mL/m²) & $61.6 \pm 43.5$ \\
\hline LVMASSI $\left(\mathrm{g} / \mathrm{m}^{2}\right)$ & $57.8 \pm 21.5$ \\
\hline
\end{tabular}

ACEl, angiotensin converting enzyme inhibitor; ARB, angiotensin receptor blocker; CMR, cardiac magnetic resonance; LVEF, left ventricular ejection fraction; LVEDVI, left ventricular end-diastolic volume index; LVESVI, left ventricular end-systolic volume index; LVMASSI, left ventricular mass index; SD, standard deviation.

using Friedman test, while CR was analyzed by repeated measures ANOVA. Intra- and inter-observer agreement was evaluated by Kappa ( $\kappa)$ analysis. A P value of less than 0.05 was considered statistical significance. The data were analyzed using SPSS Statistics version 23 (SPSS, Inc., Chicago, IL, USA).

\section{Results}

\section{Patient characteristics}

Clinical characteristics of the patients are shown in Table 2. Average age of the entire cohort at CMR exam was $66.8 \pm 10.2$ years, $30.4 \%$ female in a total of 46 patients, and mean LVEF was $48.7 \% \pm 18.8 \%$. Among 41 patients with suspected coronary artery disease, myocardial scar was identified in 30 patients (73.2\%) according to expert consensus based on findings of all LGE techniques. Detailed findings of cardiac function and perfusion were analyzed but not reported here as they are not part of this study.

\section{Scar detection and transmurality}

LGE using routine $3 \mathrm{D}$-IR, PSIR, and proposed DB method were successfully completed in all 46 patients with no imaging failures. No images were deemed nondiagnostic with significant artifact. General image quality was rated highest in DB-LGE $(3.91 \pm 0.63)$ compared to $3 \mathrm{D}$-IR $(3.51 \pm 0.76, \mathrm{P}<0.001)$ and PSIR $(3.83 \pm 0.72$, $\mathrm{P}=0.376)$. Confidence scores for scar detection were significantly higher for DB-LGE $(4.33 \pm 0.57)$ compared with 3D-IR $(3.74 \pm 0.82, \mathrm{P}<0.001)$ and PSIR $(3.97 \pm 0.78$, $\mathrm{P}=0.025)$. In general, the blood pool was completely and consistently nulled in all cases in DB-LGE. While the normal myocardium was partially nulled, scar showed hyperenhancement, which improved tissue delineation and visual inspection, in comparison to $3 \mathrm{D}-\mathrm{IR}$ and PSIR. Figure 2 exhibits case images in a series of exams with myocardial scar. In Figure $2 A$ and Figure $2 B$ slight Gibbs ringing artifact at the edge of the blood pool and myocardial wall in both 3D-IR and PSIR (arrows) was not visible in DB-LGE. In addition, aliasing artifacts that obscured scar visualization (short arrows) was also clearly not evident in the blood suppressed images.

Inter- and intra-observer agreement for scar presence was excellent for all methods $(\kappa \geq 0.87, \mathrm{P}<0.001)$. 3D-IR tended to have relatively lower inter-observer agreement for scar transmurality detection (0.652 at the patient level and 0.562 to 0.739 at the segment level), compared to PSIR (0.826 at the patient level and 0.648 to 0.727 at the segment level) and DB (0.778 at the patient level and 0.729 to 0.761 at the segment level). Details can be seen in Tables 3,4. Intra-observer agreement of LAD.

\section{Subendocardial scar assessment}

Examples of DB-LGE demonstrating better assessment 


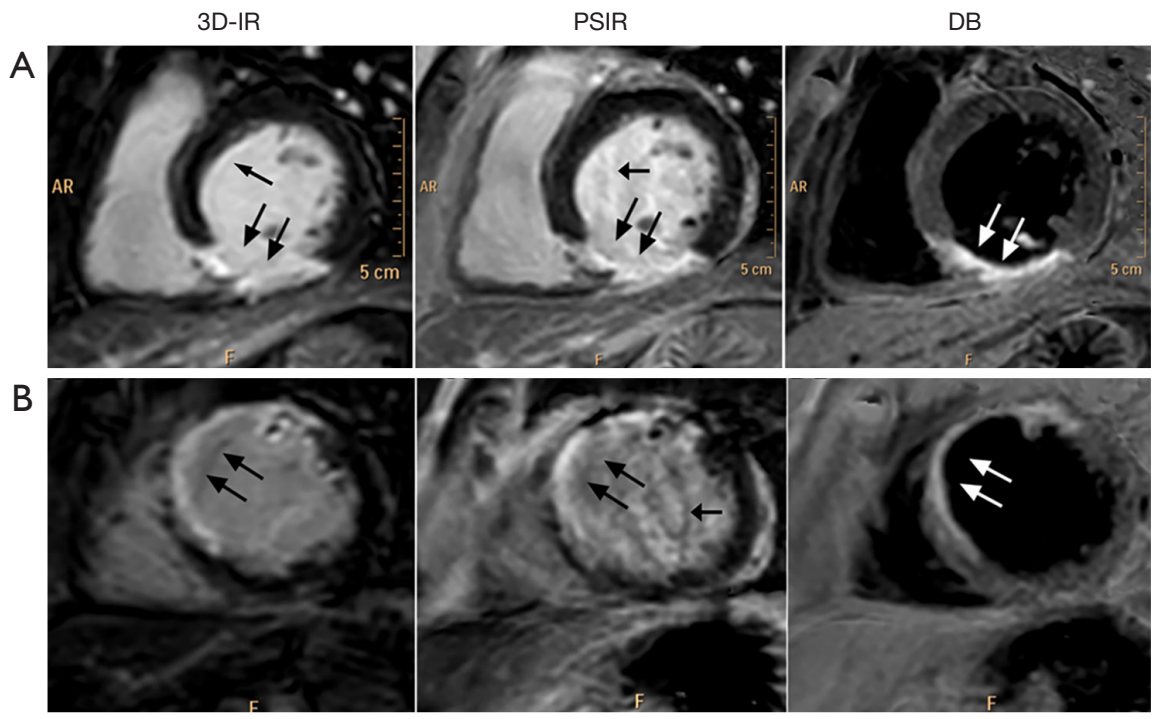

C

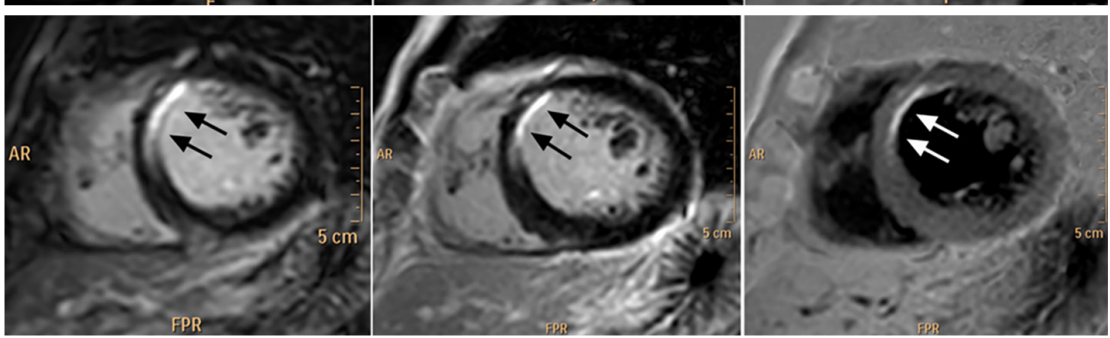

$\mathrm{D}$

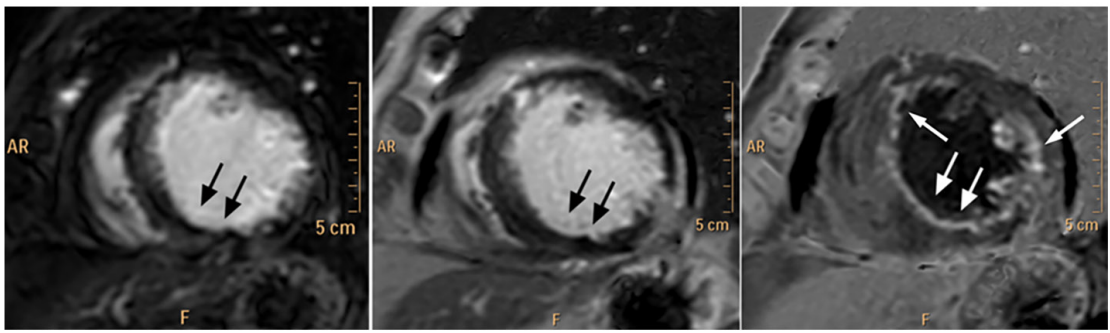

E

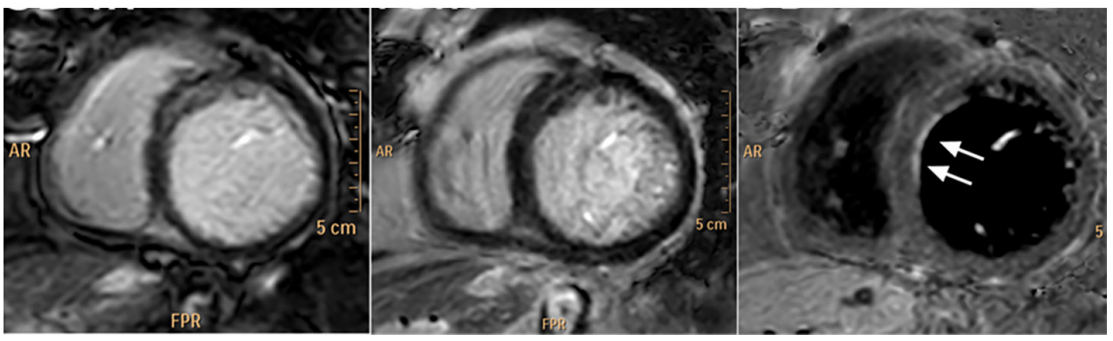

Figure 2 Typical cases of 3D-IR, PSIR and DB-LGE in patients referred to CMR for evaluation of coronary artery disease (CAD). $(\mathrm{A}, \mathrm{B})$ Fifty-three-year-old and 74-year-old male patients presenting transmural scar at the inferior wall, and anterior and anteroseptal wall, respectively. Arrows: Gibbs artifact manifested as ringing at the edge of the blood pool and myocardial wall (A,B); short arrows: inhomogeneous blood pool caused by image artifacts; white arrows: clear evidence of the scar without presence of the artifacts in DB images. (C-E) Three patients with subendocardial scar: (C) scar at the anterior and anteroseptal wall (arrows) in a 62-year-old male patient was visible in all three techniques but better delineated in DB images; (D) areas of scar were more conspicuous in DB images (white arrows) demonstrating multi-vessel involvement in a 73-year-old male patient at the inferior, inferoseptal and inferolateral wall, both with triple vessel disease; (E) scar at the anteroseptal wall was visible only in DB images (white arrows) in a 77-year-old female patient. AR, anterior right; F, foot; FPR, foot posterior right; CMR, cardiac magnetic resonance; 3D-IR, three-dimensional gradient-echo inversion recovery; DB, dark-blood; LGE, late gadolinium enhancement; PSIR, phase-sensitive inversion recovery. 
Table 3 Myocardial scar detection using 3D-IR, PSIR and DB-LGE techniques ( $n=46)$. Qualitative assessment of image quality and scar reading

\begin{tabular}{lcccc}
\hline Assessment parameters & 3D-IR & PSIR & DB & P value \\
\hline Scar presence, $\mathrm{n}(\%)$ & $33(71.73)$ & $34(73.91)$ & $35(76.08)$ & 0.223 \\
Confidence score for scar reading, mean \pm SD & $3.74 \pm 0.82$ & $3.97 \pm 0.78$ & $4.33 \pm 0.57$ & $<0.001^{\mathrm{a}, \mathrm{b}, \mathrm{c}}$ \\
Image quality, mean \pm SD & $3.51 \pm 0.76$ & $3.83 \pm 0.72$ & $3.91 \pm 0.63$ & $<0.001^{\mathrm{a}, \mathrm{b}}$ \\
\hline
\end{tabular}

${ }^{a}$, significant difference between 3D-IR and PSIR; ${ }^{b}$, significant difference between 3D-IR and DB; ${ }^{c}$, significant difference between PSIR and DB. 3D-IR, three-dimensional gradient-echo inversion recovery; PSIR, two-dimensional phase-sensitive inversion recovery; DB, dark-blood; LGE, late gadolinium enhancement; SD, standard deviation.

Table 4 Myocardial scar detection using 3D-IR, PSIR and DB-LGE techniques ( $\mathrm{n}=46)$. Intra- and inter-observer agreement was reported by Kappa analysis

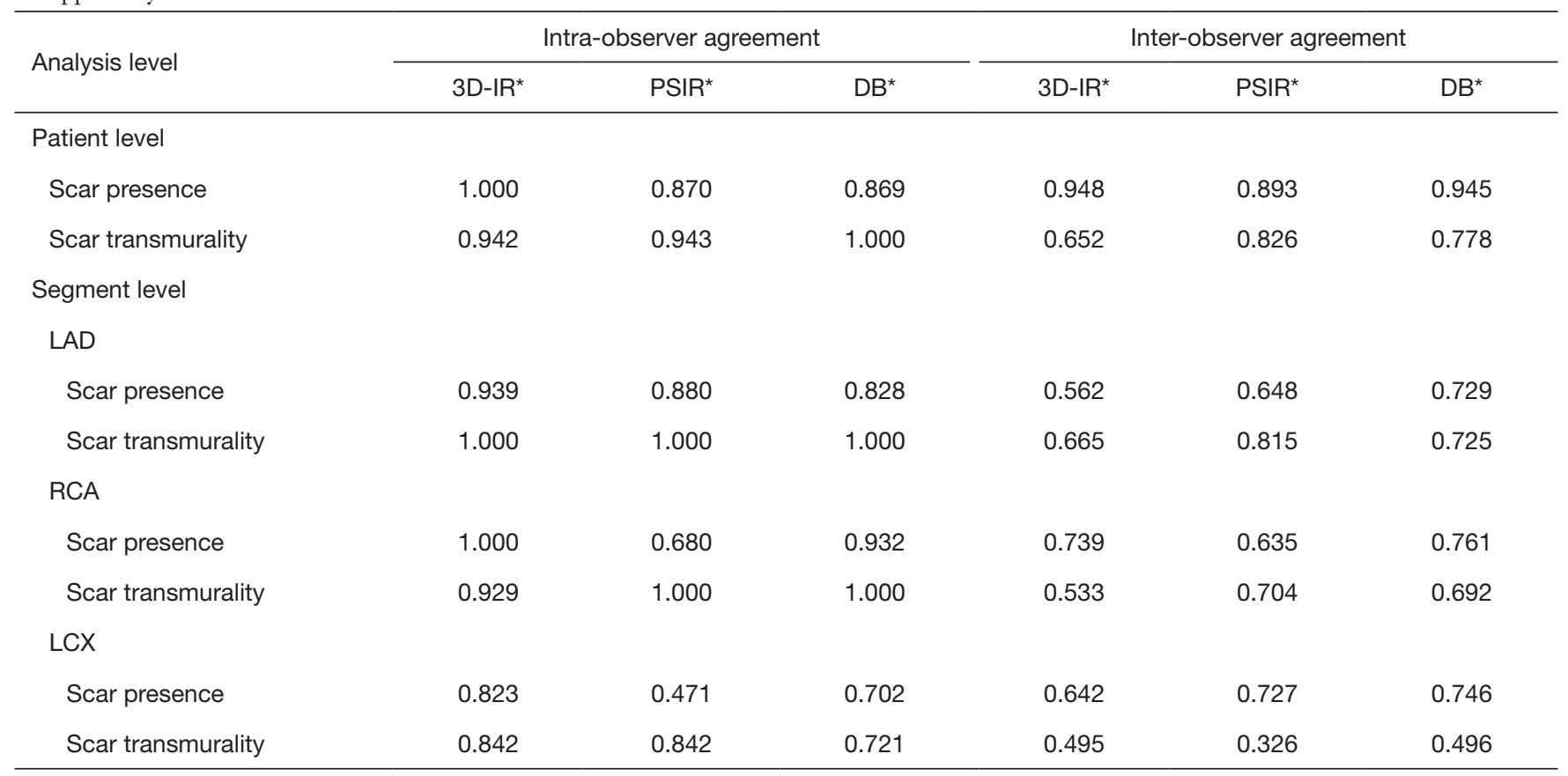

*, P values <0.001. 3D-IR, three-dimensional gradient-echo inversion recovery; PSIR, two-dimensional phase-sensitive inversion recovery; $\mathrm{DB}$, dark-blood; LGE, late gadolinium enhancement; LAD, left anterior descending coronary artery; RCA, right coronary artery; LCX, left circumflex artery.

of subendocardial scar than conventional bright-blood LGE methods are shown in Figure $2 C, 2 E$ for 3 selected patients. In Figure 2C, a subendocardial scar is clearly present at anterior and anteroseptal wall for all techniques (arrows), whereas its extent is better appreciated due to better tissue contrast and lack of image artifacts. The multivessel involvement seen in the DB images of Figure 2D is difficult to assess in the bright blood image (arrows). A subendocardial scar of the anteroseptal wall at the basal level (arrows) seen in DB-LGE in Figure 2E may have been missed entirely in the 3D-IR and PSIR images.

\section{Detection rate}

Among 30 identified scar cases according to the reader consensus ( $73.2 \%$ of 41 patients), two were missed by $3 \mathrm{D}$ IR or PSIR, both rated as minimally diagnostic with low detection confidence. Figure 3 illustrates the performance of DB and bright-blood LGE CMR for detection of myocardial infarction (MI) based upon reader consensus (41 patients, 656 segments). Overall, all techniques had a similar detection rate at the patient level, while DB had a highest rate for subendocardial scar detection (99.4\%), followed by PSIR $(61.0 \%, \mathrm{P}<0.001)$ and 3D-IR $(57.8 \%, \mathrm{P}<0.001)$. 

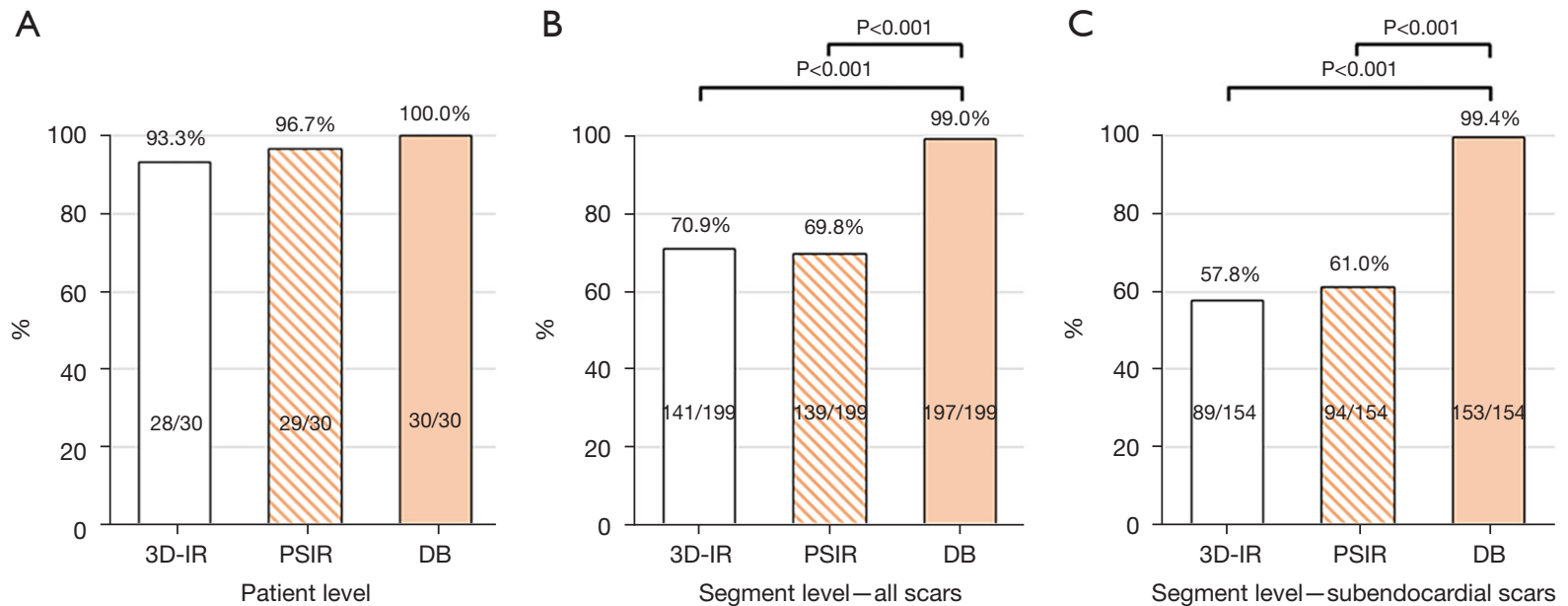

Figure 3 Scar detection rate of DB-LGE at patient and segment levels comparing to conventional bright-blood LGE using 3D-IR and PSIR. (A) Patient level. (B) Segment level of all scars. (C) Segment level of subendocardial scars. DB, dark-blood; 3D-IR, three-dimensional gradient-echo inversion recovery; LGE, late gadolinium enhancement; PSIR, phase-sensitive inversion recovery.

\section{Scar-tissue contrast}

The CR (\%) between scar and blood $\mathrm{CR}_{\text {scar-blood }}$ was significantly higher for DB $(51.9 \pm 19.8 \%$ compared with $3 \mathrm{D}$ IR $(13.1 \pm 10.6 \%), \mathrm{P}<0.001)$ and PSIR $(14.0 \pm 10.0 \%, \mathrm{P}<0.001)$. There was no significant difference for $\mathrm{CR}_{\text {scar-myo }}$ and $\mathrm{CR}_{\text {blood- }}$ myo among all three techniques (Figure 4).

\section{Discussion}

This study evaluated DB-LGE using an adapted approach based on T2-prep PSIR (15) and segmented T1-weighted gradient-echo readout with a simplified timing scheme with fixed parameters on $3 \mathrm{~T}$, with comparison to two widely used bright-blood protocols. The results demonstrate that consistent blood suppression in LV and higher scarblood contrast in DB-LGE improves scar detection in patients with known or suspected CAD, particularly in subendocardial scars, associated with higher confidence and easier interpretation. The proposed approach does not require additional scout scans, ROI measurements or prolonged acquisition, which have benefits for scan operation and clinical workflow (20).

\section{Scar visibility with dark blood}

Currently we use 3D segmented-gradient-echo inversionrecovery (3D-IR) sequence (7) in our clinical routine, which covers the entire heart within one single but relatively long breathhold. PSIR images show contrast enhancement over a wide range of values and make nulling of normal myocardium less dependent on the chosen TI, with relatively longer scan time due to alternating RR-triggered acquisition (9). So far, several methods have been proposed to increase the scar-blood contrast including double inversions $(21,22)$, magnetization transfer $(11,23)$, and T2-prepared techniques $(12,15)$. While a detailed explanation and comparison of these techniques are given in the recent review article (24), similar approaches based on T2-preparation so far $(12,15)$ require additional steps before DB-LGE including scout scan and manual ROI drawing to determine the timing parameters as well as manual entry of myocardial and blood pool T1 in sequence user interface (12-15). This process typically takes about one minute and is an added level of complexity in the clinical workflow. The approach applied in this work is adapted from the same principle using userindependent parameter setting with fixed values for $3 \mathrm{~T}$. The inversion time and T2-prep echo time were selected based on the testing results in 5 clinically confirmed CAD cases with scars at the initial phase of this study for differentiation of scar and blood contrast that share a similar T1 but different T2 values. In all patients, DB-LGE exhibited consistently nulled blood. With myocardial scar much brighter than the blood pool, it improves scar-blood contrast and more readily allows for robust scar-blood delineation. As a result, general image quality was highest in DB-LGE compared to $3 \mathrm{D}-\mathrm{IR}$ and PSIR. This also improves scar 

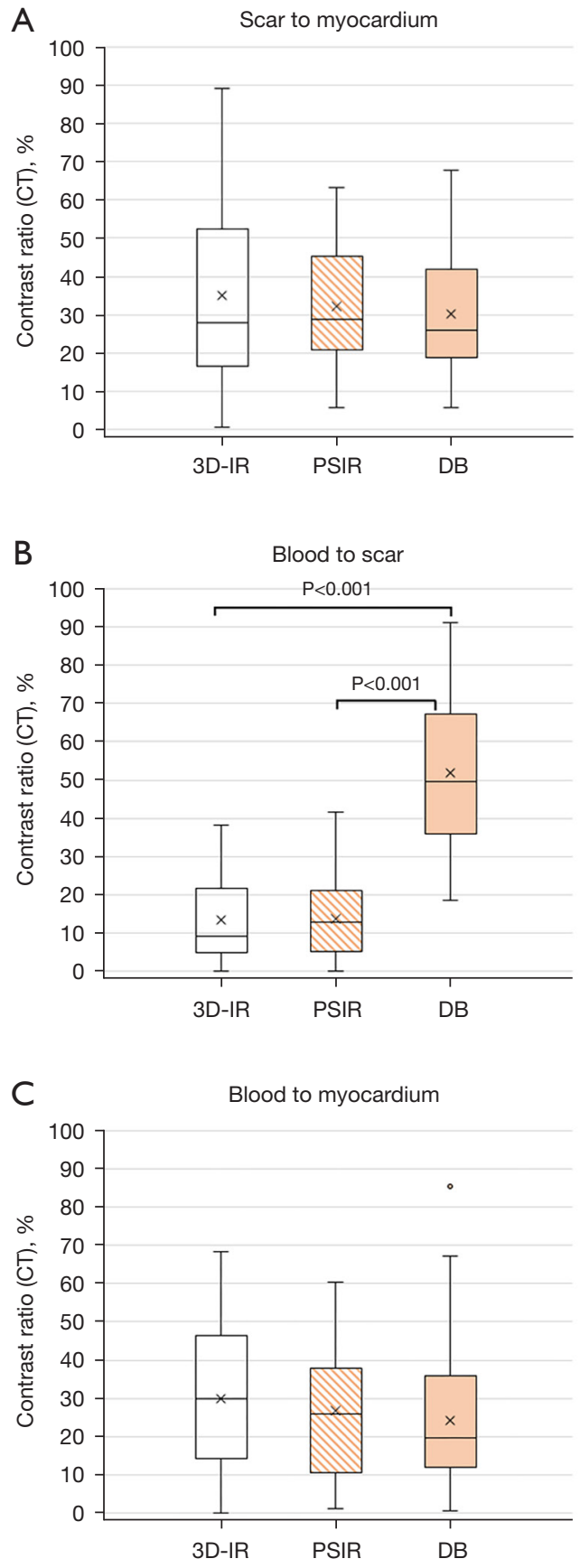

Figure 4 Tissue contrast ratio (CR) in 3D-IR, PSIR and DB-LGE CMR. (A) Contrast ratio between scar and blood $\left(\mathrm{CR}_{\text {scar-blood }}\right)$. (B) Contrast ratio between scar and normal myocardium $\left(\mathrm{CR}_{\text {scar-myo }}\right)$. $(\mathrm{C})$ Contrast ratio between blood and normal myocardium $\left(\mathrm{CR}_{\text {blood-myo }}\right)$. CMR, cardiac magnetic resonance; 3D-IR, three-dimensional inversion recovery; DB, dark-blood; LGE, late gadolinium enhancement; PSIR, phase-sensitive inversion recovery. visualization and leads to significantly higher confidence in scar detection than conventional 3D-IR and PSIR. Recently, DB-LGE techniques without additional magnetization preparation are proposed, which have achieved similar results at both 1.5 and $3 \mathrm{~T}(16,17)$. A direct comparison is not included in the present work and should be investigated in the future studies.

\section{Scar detection rate of $D B-L G E$}

Overall detection rate of myocardial scar was higher in DB-LGE comparing to the standard 3D-IR and PSIR at both patient and segment levels. This is in accordance with its highest confidence and quality rating. 3D-IR tends to interpret more scar as transmural. This is likely due to its lower spatial resolution and associated partial volume effect, particularly at areas close to the ventricular apex (25). Although PSIR has a higher spatial resolution, its similar signal enhancement degree between myocardial scar and blood pool after contrast bolus injection, is deemed as the main difficulty in detecting infarcted myocardium, particularly in the subendocardial area, as also observed in conventional IR-based LGE $(6,7,10)$. This is overcome by DB-LGE with improved scar-blood contrast, which has yielded highest detection rate $(99.4 \%$ vs. $57.8 \%$ for $3 \mathrm{D}$ IR and $61.0 \%$ for PSIR, $\mathrm{P}<0.001)$ for subendocardial scar (Figure 3). For the two cases, where no scar was seen in 3DIR or PSIR, DB-LGE revealed positive scar reading and was in consistency to the findings from CMR perfusion and coronary angiography demonstrating myocardial ischemia and significant coronary artery disease, as seen in Figure 5.

\section{Scar-tissue contrast}

Scar-to-blood contrast is deemed as one of the most important factors to interpret myocardial scar. Using CR, we have shown that DB-LGE exhibited highest value between scar and blood $(51.9 \% \pm 19.8 \%)$, significantly better than that in bright-blood LGE $(13.1 \% \pm 10.6 \%$ for $3 \mathrm{D}$-IR and $14.0 \% \pm 10.0 \%$ for PSIR, both $\mathrm{P}<0.001$, Figure 4$)$. We did not measure contrast-to-noise ratio (CNR) directly, because this requires obtaining separate noise maps in each patient to account for influence from parallel imaging or compressed sensing reconstruction $(17,26)$ which will prolong the examination times. Figure 6 shows one example of the conventional PSIR and DB images using T2-prep PSIR in 


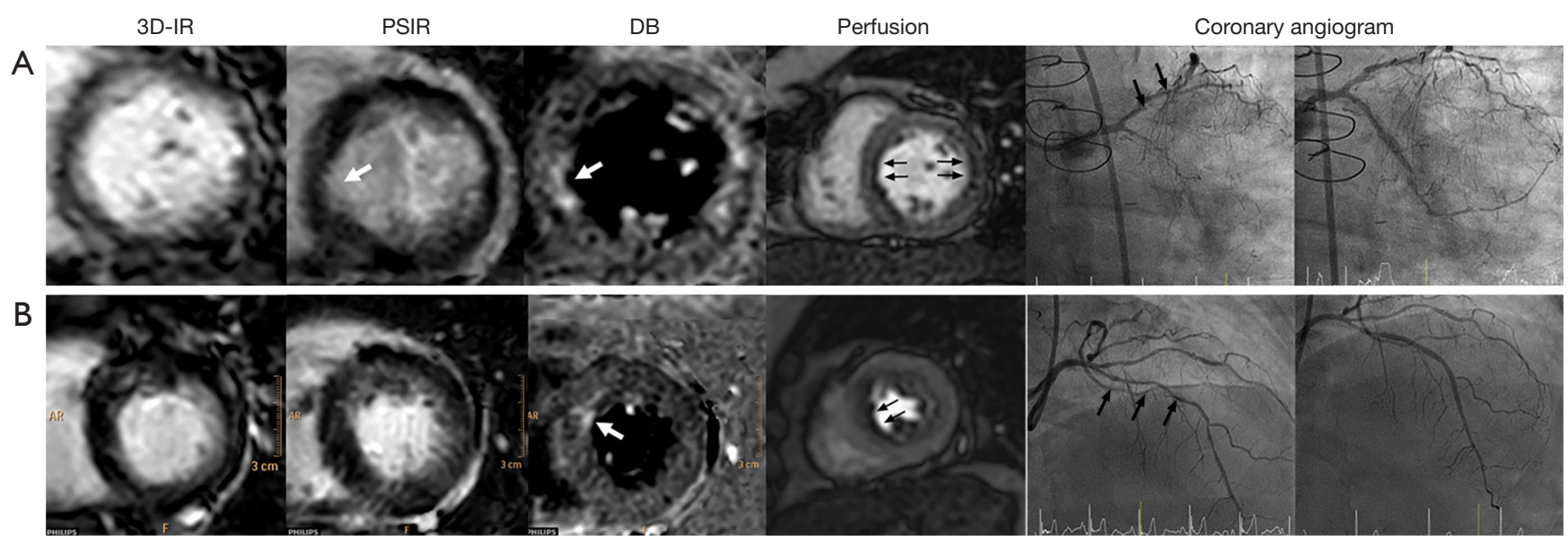

Figure 5 Myocardial scar not visible in conventional bright-blood images but detected by DB-LGE CMR. (A) Subendocardial scar at the interventricular septum in a 74-year-old female patient clearly demonstrated in the DB image and minimally visible in PSIR (arrows), while completely missed in 3D-IR. The patient had a history of coronary artery bypass graft surgery presented with a recent history of non-STsegment elevation myocardial infarction. CMR perfusion showed myocardial ischemia at anteroseptal wall and lateral wall (arrows), while coronary angiogram showed occlusion of saphenous vein graft to circumflex artery and diffuse disease of left anterior descending artery after the distal anastomosis. She underwent successful stenting at LCX, while ischemia in the anteroseptal wall was treated with medication. (B) Areas of scar at the interventricular septum in a 66-year-old female patient only evident in the DB image (arrows). The patient had a recent history of with paroxysmal atrial fibrillation and atypical chest pain. CMR perfusion showed anterior wall and anteroseptal wall ischemia (arrows), consistent with LAD coronary artery disease. The angiogram showed significant proximal LAD stenosis. PCI with drug eluting stenting was successfully performed. AR, anterior right; F, foot; CMR, cardiac magnetic resonance; 3D-IR, three-dimensional inversion recovery; DB, dark-blood; LAD, left anterior descending coronary artery; LGE, late gadolinium enhancement; LCX, left circumflex artery; PCI, percutaneous coronary intervention.

a patient with subendocardial scar. A marked difference of contrast difference between scar and blood with better border delineation to the LV cavity for DB-LGE is illustrated in the corresponding schematic plot (Figure 6B). The measured relative signal profiles referencing to normal myocardium in the same example also demonstrates a clearly improved CR between scar and blood $\left(\mathrm{CR}_{\text {scar-blood }}\right)$, as seen in Figure $6 C$. Although scar-to-myocardium and blood-to-myocardium contrasts were slightly lower in DB-LGE compared to the other bright-blood techniques, the differences were not statistically significant and this was deemed not to influence image interpretation or scar detection, as reflected by the highest qualitative scores in image quality assessment and confidence reading for DB images.

\section{Limitations}

Whilst we have derived detection rate of DB-LGE based on expert consensus, there is no histological confirmation of myocardial scar performed. While recent studies have reported an excellent correlation of DB-LGE with histopathological specimen and provided measures on diagnostic performance $(11,27)$, future studies validating the findings using the proposed method against histology would be of great value $(7,28)$, though it is difficult to achieve in a clinical setup. Scar size was evaluated as transmural extent of myocardial wall thickness and was not used as a metrics for comparison in this study. Though different methods for quantitative size measurement have been seen in the published studies $(11,12,26)$, there is no consensus and one cannot verify which method is more accurate. We did not study the effect of arrhythmia or fat signal or cardiac implants on DB-LGE image quality. While all patients in our study were in sinus rhythm during the examination, fat signal with similar $\mathrm{T} 1$ relaxation time to scar could potentially lead to false positive and further investigation incorporating robust fat saturation or separation is needed. For patients with pacemakers or implantable defibrillators, a wideband version of this LGE sequence could help to mitigate potential off-resonance artifacts and would be of 


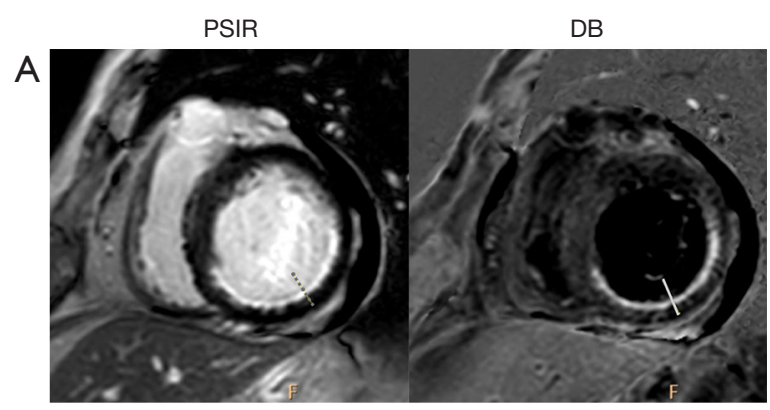

B
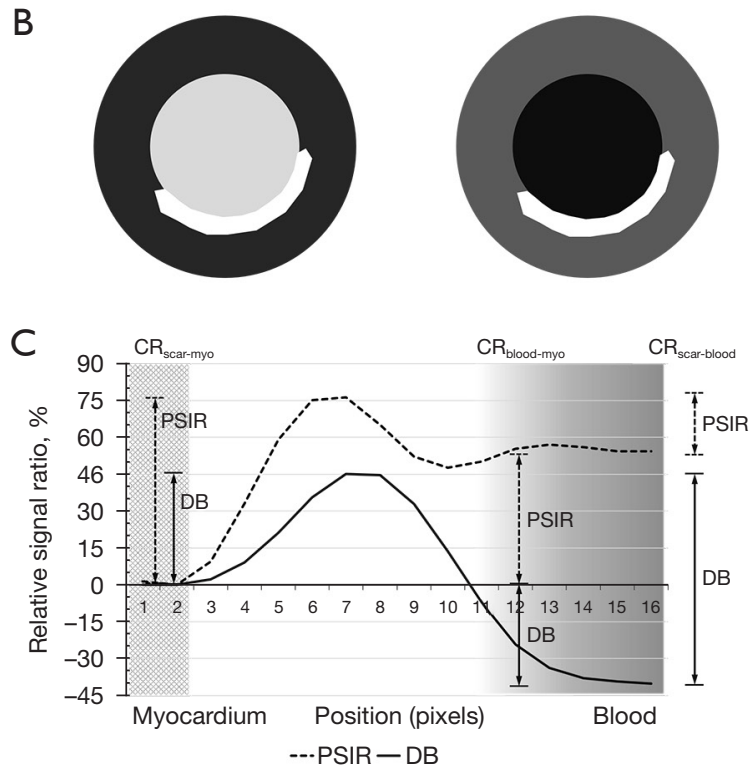

Figure 6 An illustrative case with comparison between bright-blood PSIR and DB-LGE CMR for detection of subendocardial scar. (A) Typical image appearance with presence of a subendocardial scar at the inferior and inferolateral wall in the same patient. (B) Simplified schematic diagram based on A demonstrating visual perception of tissue contrast. (C) Relative signal ratio profiles normalized to myocardium showing clearly improved scar-blood contrast ratio $\left(\mathrm{CR}_{\text {scar-blood }}\right)$ for DB (straight) compared with bright-blood (dotted) PSIR, though myocardium-blood $\left(\mathrm{CR}_{\text {blood-myo }}\right)$ and myocardium-scar contrast ratios $\left(\mathrm{CR}_{\text {scar-myo }}\right)$ were slighted lower for DB compared with PSIR (details see text). For calculation of the signal ratio and contrast ratio, the manufacturer's applied scaling in the stored DICOM data was removed by converting the data to floating point values as this reflects the true MR signal range directly after reconstruction. No additional scaling was applied for comparison between the LGE methods. CMR, cardiac magnetic resonance; DB, dark-blood; LGE, late gadolinium enhancement; PSIR, phase-sensitive inversion recovery.

interest in the future. DB images were only acquired in the short-axis planes, as was the case for 3D-IR that is currently routinely used in our hospital. Nevertheless, it would be interesting to observe DB-LGE in the other cardiac planes for scar detection and delineation. Last but not least, fixed timing parameters were applied for the DB-LGE approach in this work. These were weighted to prioritize nulling the LV blood pool based on clinically confirmed scars on $3 \mathrm{~T}$ at the initial phase of this study. This might lead to suboptimal blood suppression in certain cases, such as in the right ventricle (RV) cavity, where T1 of the deoxygenated blood is shorter than the LV blood (15). In particular, the selection of the timing parameters was not to achieve specific scarblood ratio, similar as in the other proposed methods $(11-17,23,25,28)$. This could be done via patient-specific, data-driven or even machine learning-aided optimization (20), in which case full automation may be expected to address challenge in routine clinical workflow and to minimize additional examination times. Given these limitations, larger studies in patients are warranted to further assess 
the diagnostic value and clinical impact of DB-LGE for scar detection, particularly in comparison to other existing methods either without additional magnetization preparation $(16,17)$ or in free-breathing $(29)$ or $3 \mathrm{D}$ imaging $(30)$.

\section{Conclusions}

In patients with suspected CAD, DB-LGE results in higher confidence in myocardial scar detection with increased overall image quality on $3 \mathrm{~T}$. This approach allows improved scar detection, particularly in detecting subendocardial scars, compared to the current standard bright-blood methods, and thus may be of potential value in patient management of coronary artery disease. Future comparison to other available techniques in large clinical cohorts are warranted.

\section{Acknowledgments}

The authors thank Ahthit Yindeengam for statistical analysis and data management.

Funding: None.

\section{Footnote}

Reporting Checklist: The authors have completed the STARD reporting checklist. Available at https://dx.doi. org/10.21037/qims-21-704

Conflicts of Interest: All authors have completed the ICMJE uniform disclosure form (available at https://dx.doi. org/10.21037/qims-21-704). SZ reports that he is an employee of Philips. The other authors have no conflicts of interest to declare.

Ethical Statement: The authors are accountable for all aspects of the work in ensuring that questions related to the accuracy or integrity of any part of the work are appropriately investigated and resolved. The study was conducted in accordance with the Declaration of Helsinki (as revised in 2013). This study was approved by the Siriraj Institutional Review Board (SIRB) of the Faculty of Medicine Siriraj Hospital, Mahidol University, Bangkok, Thailand. All patients provided written informed consent prior to participation.

Open Access Statement: This is an Open Access article distributed in accordance with the Creative Commons
Attribution-NonCommercial-NoDerivs 4.0 International License (CC BY-NC-ND 4.0), which permits the noncommercial replication and distribution of the article with the strict proviso that no changes or edits are made and the original work is properly cited (including links to both the formal publication through the relevant DOI and the license). See: https://creativecommons.org/licenses/by-nc-nd/4.0/.

\section{References}

1。 Klem I, Heitner JF, Shah DJ, Sketch MH Jr, Behar V, Weinsaft J, Cawley P, Parker M, Elliott M, Judd RM, Kim RJ. Improved detection of coronary artery disease by stress perfusion cardiovascular magnetic resonance with the use of delayed enhancement infarction imaging. J Am Coll Cardiol 2006;47:1630-8.

2. Krittayaphong R, Maneesai A, Chaithiraphan V, Saiviroonporn $\mathrm{P}$, Chaiphet O, Udompunturak S. Comparison of diagnostic and prognostic value of different electrocardiographic criteria to delayed-enhancement magnetic resonance imaging for healed myocardial infarction. Am J Cardiol 2009;103:464-70.

3. Bruder O, Wagner A, Lombardi M, Schwitter J, van Rossum A, Pilz G, et al. European Cardiovascular Magnetic Resonance (EuroCMR) registry--multi national results from 57 centers in 15 countries. J Cardiovasc Magn Reson 2013;15:9.

4. El Aidi H, Adams A, Moons KG, Den Ruijter HM, Mali WP, Doevendans PA, Nagel E, Schalla S, Bots ML, Leiner T. Cardiac magnetic resonance imaging findings and the risk of cardiovascular events in patients with recent myocardial infarction or suspected or known coronary artery disease: a systematic review of prognostic studies. J Am Coll Cardiol 2014;63:1031-45.

5. Kim RJ, Judd RM, Chen EL, Fieno DS, Parrish TB, Lima JA. Relationship of elevated $23 \mathrm{Na}$ magnetic resonance image intensity to infarct size after acute reperfused myocardial infarction. Circulation 1999;100:185-92.

6. Edelman RR. Contrast-enhanced MR imaging of the heart: overview of the literature. Radiology 2004;232:653-68.

7. Kim RJ, Fieno DS, Parrish TB, Harris K, Chen EL, Simonetti O, Bundy J, Finn JP, Klocke FJ, Judd RM. Relationship of MRI delayed contrast enhancement to irreversible injury, infarct age, and contractile function. Circulation 1999;100:1992-2002.

8. Attili AK, Schuster A, Nagel E, Reiber JH, van der Geest RJ. Quantification in cardiac MRI: advances in image acquisition and processing. Int $\mathrm{J}$ Cardiovasc Imaging 
2010;26 Suppl 1:27-40.

9. Kellman P, Arai AE, McVeigh ER, Aletras AH. Phasesensitive inversion recovery for detecting myocardial infarction using gadolinium-delayed hyperenhancement. Magn Reson Med 2002;47:372-83.

10. Kim RJ, Albert TS, Wible JH, Elliott MD, Allen JC, Lee JC, Parker M, Napoli A, Judd RM; Gadoversetamide Myocardial Infarction Imaging Investigators. Performance of delayed-enhancement magnetic resonance imaging with gadoversetamide contrast for the detection and assessment of myocardial infarction: an international, multicenter, double-blinded, randomized trial. Circulation 2008;117:629-37.

11. Kim HW, Rehwald WG, Jenista ER, Wendell DC, Filev P, van Assche L, Jensen CJ, Parker MA, Chen EL, Crowley ALC, Klem I, Judd RM, Kim RJ. Dark-Blood Delayed Enhancement Cardiac Magnetic Resonance of Myocardial Infarction. JACC Cardiovasc Imaging 2018;11:1758-69.

12. Francis R, Kellman P, Kotecha T, Baggiano A, Norrington K, Martinez-Naharro A, Nordin S, Knight DS, Rakhit RD, Lockie T, Hawkins PN, Moon JC, Hausenloy DJ, Xue H, Hansen MS, Fontana M. Prospective comparison of novel dark blood late gadolinium enhancement with conventional bright blood imaging for the detection of scar. J Cardiovasc Magn Reson 2017;19:91.

13. Basha TA, Tang MC, Tsao C, Tschabrunn CM, Anter E, Manning WJ, Nezafat R. Improved dark blood late gadolinium enhancement (DB-LGE) imaging using an optimized joint inversion preparation and T2 magnetization preparation. Magn Reson Med 2018;79:351-60.

14. Fahmy AS, Neisius U, Tsao CW, Berg S, Goddu E, Pierce P, Basha TA, Ngo L, Manning WJ, Nezafat R. Gray blood late gadolinium enhancement cardiovascular magnetic resonance for improved detection of myocardial scar. J Cardiovasc Magn Reson 2018;20:22.

15. Kellman P, Xue H, Olivieri LJ, Cross RR, Grant EK, Fontana M, Ugander M, Moon JC, Hansen MS. Dark blood late enhancement imaging. J Cardiovasc Magn Reson 2016;18:77.

16. Holtackers RJ, Van De Heyning CM, Nazir MS, Rashid I, Ntalas I, Rahman H, Botnar RM, Chiribiri A. Clinical value of dark-blood late gadolinium enhancement cardiovascular magnetic resonance without additional magnetization preparation. J Cardiovasc Magn Reson 2019;21:44.

17. Holtackers RJ, Chiribiri A, Schneider T, Higgins DM, Botnar RM. Dark-blood late gadolinium enhancement without additional magnetization preparation. J Cardiovasc Magn Reson 2017;19:64.

18. Cerqueira MD, Weissman NJ, Dilsizian V, Jacobs AK, Kaul S, Laskey WK, Pennell DJ, Rumberger JA, Ryan T, Verani MS; American Heart Association Writing Group on Myocardial Segmentation and Registration for Cardiac Imaging. Standardized myocardial segmentation and nomenclature for tomographic imaging of the heart. A statement for healthcare professionals from the Cardiac Imaging Committee of the Council on Clinical Cardiology of the American Heart Association. Circulation 2002;105:539-42.

19. Dietrich O, Raya JG, Reeder SB, Reiser MF, Schoenberg SO. Measurement of signal-to-noise ratios in MR images: influence of multichannel coils, parallel imaging, and reconstruction filters. J Magn Reson Imaging 2007;26:375-85.

20. Polacin M, Gastl M, Kapos I, Eberhard M, Weber L, Gotschy A, von Spiczak J, Kozerke S, Alkadhi H, Manka R. Novel Magnetic Resonance Late Gadolinium Enhancement With Fixed Short Inversion Time in Ischemic Myocardial Scars. Invest Radiol 2020;55:445-50.

21. Song HK, Wright AC, Wolf RL, Wehrli FW. Multislice double inversion pulse sequence for efficient black-blood MRI. Magn Reson Med 2002;47:616-20.

22. Foo TK, Wolff SD, Gupta SN, Kraitchman DL. Enhanced viability imaging: improved contrast in myocardial delayed enhancement using dual inversion time subtraction. Magn Reson Med 2005;53:1484-9.

23. Muscogiuri G, Rehwald WG, Schoepf UJ, Suranyi P, Litwin SE, De Cecco CN, Wichmann JL, Mangold S, Caruso D, Fuller SR, Bayer Nd RR, Varga-Szemes A. T(Rho) and magnetization transfer and INvErsion recovery (TRAMINER)-prepared imaging: A novel contrast-enhanced flow-independent dark-blood technique for the evaluation of myocardial late gadolinium enhancement in patients with myocardial infarction. J Magn Reson Imaging 2017;45:1429-37.

24. Holtackers RJ, Van De Heyning CM, Chiribiri A, Wildberger JE, Botnar RM, Kooi ME. Dark-blood late gadolinium enhancement cardiovascular magnetic resonance for improved detection of subendocardial scar: a review of current techniques. J Cardiovasc Magn Reson 2021;23:96.

25. Turkbey EB, Nacif MS, Noureldin RA, Sibley CT, Liu S, Lima JA, Bluemke DA. Differentiation of myocardial scar from potential pitfalls and artefacts in delayed enhancement MRI. Br J Radiol 2012;85:e1145-54. 
26. Foley JRJ, Broadbent DA, Fent GJ, Garg P, Brown LAE, Chew PG, Dobson LE, Swoboda PP, Plein S, Higgins DM, Greenwood JP. Clinical evaluation of two dark blood methods of late gadolinium quantification of ischemic scar. J Magn Reson Imaging 2019;50:146-52.

27. Holtackers RJ, Gommers S, Heckman LIB, Van De Heyning CM, Chiribiri A, Prinzen FW. Histopathological Validation of Dark-Blood Late Gadolinium Enhancement MRI Without Additional Magnetization Preparation. J Magn Reson Imaging 2021. [Epub ahead of print]. doi: 10.1002/jmri.27805.

28. Liu CY, Wieben O, Brittain JH, Reeder SB. Improved delayed enhanced myocardial imaging with T2-Prep inversion recovery magnetization preparation. J Magn

Cite this article as: Krittayaphong $\mathrm{R}$, Zhang $\mathrm{S}$, Tanapibunpon $\mathrm{P}$, Kaolawanich Y, Nakyen S. Dark-blood late gadoliniumenhancement cardiac magnetic resonance imaging for myocardial scar detection based on simplified timing scheme: single-center experience in patients with suspected coronary artery disease. Quant Imaging Med Surg 2022;12(2):1037-1050. doi: 10.21037/qims-21-704
Reson Imaging 2008;28:1280-6.

29. Piehler KM, Wong TC, Puntil KS, Zareba KM, Lin K, Harris DM, Deible CR, Lacomis JM, CzeydaPommersheim F, Cook SC, Kellman P, Schelbert EB. Free-breathing, motion-corrected late gadolinium enhancement is robust and extends risk stratification to vulnerable patients. Circ Cardiovasc Imaging 2013;6:423-32.

30. Correia T, Ginami G, Rashid I, Nordio G, Hajhosseiny R, Ismail TF, Neji R, Botnar RM, Prieto C. Accelerated highresolution free-breathing $3 \mathrm{D}$ whole-heart $\mathrm{T} 2$-prepared black-blood and bright-blood cardiovascular magnetic resonance. J Cardiovasc Magn Reson 2020;22:88. 